\title{
NORMAL CONGRUENCES AND QUADRUPLY INFINITE SYSTEMS OF CURVES IN SPACE*
}

BY

JESSE DOUGLAS

INTRODUCTION

The present paper sets into relation with one another the following two geometric configurations:

First, the quadruply infinite system of curves in space, as defined by the set of differential equationst

(1)

$$
y^{\prime \prime}=F\left(x, y, z, y^{\prime}, z^{\prime}\right)
$$

$$
z^{\prime \prime}=G\left(x, y, z, y^{\prime}, z^{\prime}\right)
$$

Second, the normal congruence - a congruence of curves being any system of $\infty^{2}$ curves so distributed that one and only one passes through each point of some region of space, and a normal congruence one relative to which a system of $\infty^{1}$ orthogonal surfaces can be constructed. The congruence defined by the differential equations

$$
\frac{d x}{A(x, y, z)}=\frac{d y}{B(x, y, z)}=\frac{d z}{C(x, y, z)}
$$

is normal when and only when the Pfaffian equation

$$
A d x+B d y+C d z=0
$$

is exact-thus a congruence is in general not normal.

The simplest example of a system of $\infty^{4}$ curves in space is presented by the straight lines, one of the important occurrences of which is as the extremals of the calculus of variations problem $\int d s=$ minimum.

* Presented to the Society, February 25, 1922 (Part I) and April 28, 1923 (Part II).

$\dagger$ The functions $F$ and $G$ are assumed to be uniform and analytic in a certain region of the $x, y, z, y^{\prime}, z^{\prime}$ continuum, within which region we always remain. 
Consider the straight lines orthogonal to an arbitrary surface $\Sigma$. They are orthogonal not only to $\Sigma$ but to its $\infty^{1}$ parallel surfaces, obtained by laying off along the lines from their points of normality to $\Sigma$ constant lengths, that is, constant $\int d s$.

This property of the extremals of $\int d s=$ minimum generalizes to the extremals of any variation problem of the form

$$
\int F(x, y, z) d s=\text { minimum. }
$$

This is because for integrals of that form (and for no others) the relation of transversality in the sense of the calculus of variations reduces to orthogonality. Therefore, according to the classic theory, if we select from the $\infty^{4}$ extremals the $\infty^{2}$ that meet an arbitrary surface $\Sigma$ orthogonally, and lay off along each an arc starting from $\Sigma$ so that $\int F(x, y, z) d s$ over the arc has a fixed value, the locus of the end points is a surface orthogonal to the $\infty^{2}$ extremals. By variation of the fixed value, $\infty^{1}$ such orthogonal surfaces result.

A system of $\infty^{4}$ curves in space identifiable with the totality of extremals of a problem of the form (2) is known in the literature as a natural family, ${ }^{*}$ the name referring to the many physical interpretations. The Euler-Lagrange equations of (2) are

$$
\begin{aligned}
& y^{\prime \prime}=\left(L_{y}-y^{\prime} L_{x}\right)\left(1+{y^{\prime}}^{2}+z^{\prime 2}\right), \\
& z^{\prime \prime}=\left(L_{z}-z^{\prime} L_{x}\right)\left(1+{y^{\prime}}^{2}+z^{\prime 2}\right),
\end{aligned}
$$

where $L=\log F$, and is thus any function of $x, y, z$. The form of these differential equations may be taken as the criterion for a natural family.

The above stated property of the extremals of $\int F(x, y, z) d s$ may be formulated as follows: in a natural family the $\infty^{2}$ curves orthogonal to an arbitrary surface form a normal congruence. This theorem is due to Thomson and Tait, who stated it in a dynamical form.t

In a paper of $1910, \ddagger \mathrm{E}$. Kasner dealt with a number of geometrical problems arising from the theorem of Thomson and Tait, proving, in particular, that

* After E. Kasner. See his Differential-geometric aspects of dynamics, Princeton Colloquium Lectures, 1912, § 28.

$\dagger$ Natural Philosophy (edition of 1879), Part I, § 332 . In its optical form, the theorem is known as that of Malus; see Hadamard, Leģons sur le Calcul des Variations, p. 150.

$\ddagger$ The theorem of Thomson and Tait and natural families of trajectories, these Transactions, vol. 11 (1910), pp. 121-140. 
the property of the theorem is characteristic of natural families. The writer's attention was directed to this field of investigation by Professor Kasner, the problems of the present paper being suggested by the paper just cited. Our method of analysis is distinguished by the systematic use of equations of variation.

Our paper falls into two parts. The first determines all the possibilities as to the number of normal congruences-in the sense of the number of parameters or of arbitrary functions involved-that may be contained in a quadruply infinite system of curves in space. The second deals with special base manifolds $\Sigma$, solving the four problems of determining all families of $\infty^{4}$ space curves, such that a normal congruence is formed by (1) the curves of the family passing through an arbitrary point, (2) the curves orthogonal to an arbitrary plane, (3) the curves orthogonal to an arbitrary sphere, (4) the curves orthogonal to an arbitrary straight line.

Given a family of $\infty^{4}$ curves in space, every surface $\Sigma$ determines a congruence of curves within the family, consisting namely of the $\infty^{2}$ curves of the family orthogonal to $\Sigma$. In the case of a natural family, all these congruence have the normal character. At the other extreme is the general family of $\infty^{4}$ curves which contains no normal congruences whatever. The investigations that follow bring to light a number of intermediate types, resulting in the following complete classification.

1. The natural families, which are such that the congruence determined by an arbitrary surface $\Sigma$ is normal.

2. The quasi-natural families. These are such that all and only the surfaces $\Sigma$ which obey a certain linear partial differential equation of the second order

$$
\mathfrak{B}_{1} r+\mathfrak{B}_{z} s+\mathfrak{B}_{3} t+\mathfrak{B}_{4}=0
$$

give rise to normal congruences. The coefficients are functions of $x, y, z, p, q$ whose form depends on the particular family.

3. A type in which, if there is given arbitrarily a space curve together with a surface element belonging to the curve, there exists a unique surface $\boldsymbol{\Sigma}$ containing curve and element and determining a normal congruence.

4. A type containing $\infty^{3}$ normal congruences, so distributed that each curve of the family belongs to $\infty^{1}$ of them.

5. A type containing $\infty^{2}$ normal congruences, so distributed that each curve of the family belongs to at least one of them.

6. The normal congruences may be infinite in number, but confined to a triply infinite sub-family. In a triply infinite curve family in space the directions of the curves through an arbitrary point form a cone. It follows that in order that a surface $\Sigma$ be met by $\infty^{2}$ curves of the family orthogonally, 
it must satisfy a certain partial differential equation of the first order. Either all these surfaces give normal congruences, or else $\infty^{2}$, or $\infty^{1}$, or none of them; so that, since each normal congruence absorbs $\infty^{1}$ surfaces $\Sigma$, there are, if more than a finite number, either $\infty^{\infty}$ or $\infty^{1}$ normal congruences in the family.

7. A finite number or no normal congruences.

The base manifold $\Sigma$ may reduce to a curve or to a point. In the paper cited, Professor Kasner proved it sufficient to characterize a system of $\infty^{4}$ curves as of the natural type that the curves belonging to it which meet an arbitrary base curve orthogonally form a normal congruence. In fact, he proved that it suffices to demand this property of only the $\infty^{6}$ circles of space.

Relative to the case where the base manifold is a point, Professor Kasner showed by means of the example

$$
y^{\prime \prime}={y^{\prime}}^{2}, \quad z^{\prime \prime}=0
$$

that non-natural families exist in which the curves through an arbitrary point of space form a normal congruence*, and formulated but left unsolved the problem of determining all such families, with the remark that the direct attack on the problem by the methods of his paper would lead to great difficulties.

The second part of the present paper solves this problem together with the analogous ones relative to the plane, sphere, and straight line. The results are as follows.

1. There are three types with the normality property with respect to points: the natural, the quasi-natural, and a third type defined by a certain system of four partial differential equations in $F$ and $G$ (the equations (43), §8).

2. For the plane as base manifold, the analysis yields five possibilities, of three of which we have established the effective existence. Two of these are the natural and quasi-natural types.

3. The congruence determined by an arbitrary sphere is normal when and only when the family is natural or quasi-natural. This result affords the neatest characterization of a quasi-natural family: one in which the curves orthogonal to any sphere form a normal congruence, but where the same is not true of an arbitrary surface. It is sufficient to demand the normality property of arbitrary points and planes.

4. If the curves orthogonal to an arbitrary straight line form a normal congruence, the family is necessarily natural. It is sufficient to demand this

\footnotetext{
* The example cited is arrived at analytically. A more general example, based on geometrical considerations, may be obtained by allowing the moulure surface $M$ mentioned in $\S 6$ of the present paper to reduce, as it can, to an arbitrary point.
} 
property of any triple complex of straight lines (three lines in each pencil), or of a triple complex of curves of any kind. In fact, if the two complexes of straight lines parallel to two of the coördinate planes, respectively, give rise to normal congruences, the family must be natural.

In this way, $2 \infty^{3}$ tests as to the possession of the normality property are sufficient to decide as to the natural or non-natural character of a given system of $\infty^{4}$ curves. An interesting problem is that of finding a minimum number of tests necessary and sufficient for this purpose,-it is certain that this minimum number is either $2 \infty^{3}$ or $1 \infty^{3}$, but the calculations required to establish a proof for either case seem too complicated. We can be sure, however, that if $1 \infty^{3}$ base curves do suffice, they cannot be any whatever, for the family

$$
y^{\prime \prime}=\frac{y^{\prime}\left(1+y^{\prime 2}+z^{\prime 2}\right)}{x+z z^{\prime}}, \quad z^{\prime \prime}=0
$$

-consisting of the $\infty^{4}$ circles whose axes intersect the axis of $y$, assumed vertical, at right angles - which family is not natural (or even quasi-natural), is such that the $1 \infty^{3}$ horizontal straight lines give rise to normal congruences.

There remain these two possibilities as to which we have not been able to decide: (1) perhaps an arbitrary system of $2 \infty^{3}$ curves giving rise to normal congruences will characterize a family as natural; (2) perhaps $1 \infty^{3}$ curves are sufficient, provided they are not of a special type.

\section{Part I}

CLASSIFICATION OF QUADRUPLY INFINITE SYSTEMS OF CURVES IN SPACE WITH RESPECT TO THE NORMAL CONGRUENCES CONTAINED WITHIN THEM

\section{Equations of variation}

Our analysis being based on equations of variation, we develop the necessary formulas immediately in order to avoid later digression.

In the four-parameter family of curves defined by the equations (1) let

$$
\begin{aligned}
& y=\varphi(x ; \alpha, \beta), \\
& z=\psi(x ; \alpha, \beta)
\end{aligned}
$$

represent any two-parameter sub-family. Then we must have identically in $x, \alpha, \beta$

$$
\begin{aligned}
& \varphi^{\prime \prime}=F\left(x, \varphi, \psi, \varphi^{\prime}, \psi^{\prime}\right), \\
& \psi^{\prime \prime}=G\left(x, \varphi, \psi, \varphi^{\prime}, \psi^{\prime}\right),
\end{aligned}
$$


where the accents denote partial differentiation with respect to $x$. Differentiating these identities with respect to $\alpha$, and taking account of the commutativity of the differentiations with respect to $x$ and $\alpha$, we get

$$
\begin{aligned}
\left(\varphi_{\alpha}\right)^{\prime \prime} & =F_{y}\left(\varphi_{\alpha}\right)+F_{z}\left(\psi_{\alpha}\right)+F_{y^{\prime}}\left(\varphi_{\alpha}\right)^{\prime}+F_{z^{\prime}}\left(\psi_{\alpha}\right)^{\prime} \\
\left(\psi_{\alpha}\right)^{\prime \prime} & =G_{y}\left(\varphi_{\alpha}\right)+G_{z}\left(\psi_{\alpha}\right)+G_{y^{\prime}}\left(\varphi_{\alpha}\right)^{\prime}+G_{z^{\prime}}\left(\psi_{\alpha}\right)^{\prime}
\end{aligned}
$$

Thus the parametric derivatives $\varphi_{\alpha}, \psi_{\alpha}$ obey a system of linear differential equations. Evidently, the same equations are obeyed by $\varphi_{\beta}, \psi_{\beta}$.

\section{CONDITIONS FOR A NORMAL CONGRUENCE}

Any congruence of curves is definable by differential equations of the form

$$
\frac{d x}{A(x, y, z)}=\frac{d y}{B(x, y, z)}=\frac{d z}{C(x, y, z)}
$$

A system of $\infty^{1}$ surfaces orthogonal to the congruence exists when and only when the corresponding Pfaffian equation

$$
A d x+B d y+C d z=0
$$

is exact; for this the necessary and sufficient condition is

$$
A\left(C_{y}-B_{z}\right)+B\left(A_{z}-C_{x}\right)+C\left(B_{x}-A_{y}\right)=0 .
$$

The equations (4), assigning to each point of space a determinate direction, define a field of $\infty^{3}$ lineal elements wherefrom the congruence of curves arises by integration. The most general way of representing such an element field is by writing the five coördinates $x, y, z, y^{\prime}, z^{\prime}$ of a lineal element as functions of three parameters $t, u, v$. The condition that an element field so defined correspond to a normal congruence is

$$
\left(y^{\prime} x y\right)-\left(z^{\prime} z x\right)+y^{\prime}\left(z^{\prime} y z\right)-z^{\prime}\left(y^{\prime} y z\right)=0
$$

where the parentheses denote jacobians with respect to $t, u, v$.

3. The congruence Determined by an arbitrary base surface

Consider the general family $\mathfrak{F}$ of $\infty^{4}$ curves,

$$
\begin{aligned}
y^{\prime \prime} & =F\left(x, y, z, y^{\prime}, z^{\prime}\right), \\
z^{\prime \prime} & =G\left(x, y, z, y^{\prime}, z^{\prime}\right) .
\end{aligned}
$$


Let $\boldsymbol{\Sigma}$ designate an arbitrary surface, whose equation we write in the form

$$
x=-\varphi(y, z) \text {. }
$$

For the derivatives of $\varphi$ we shall employ the Monge notation

$$
p=\varphi_{y}, \quad q=\varphi_{z} ; \quad r=\varphi_{y y}, \quad s=\varphi_{y z}, \quad t=\varphi_{z z} .
$$

We have for the direction $1: y^{\prime}: z^{\prime}$ normal to $\Sigma$ at the point $(x, y, z)$ of $\Sigma$

$$
y^{\prime}=p, \quad z^{\prime}=q
$$

The point and direction $x, y, z, y^{\prime}=p, z^{\prime}=q$ determine in the family $\mathfrak{F}$ a unique curve $C$, whose equations can be written explicitly in the form of power series; these begin

$$
Y=y+p(X-x)+\frac{1}{2} F(X-x)^{2}+\cdots,
$$

$$
Z=z+q(X-x)+\frac{1}{2} G(X-x)^{2}+\cdots
$$

Here $X, Y, Z$ are the coördinates of a point current along $C$, and the substitutions

$$
x=-\varphi(y, z), \quad p=\varphi y(y, z), \quad q=\varphi_{z}(y, z)
$$

are to be made throughout, so that the symbol $F$, for instance, is an abbreviation for

$$
F\left[-\varphi(y, z), y, z, \varphi_{y}(y, z), \varphi_{z}(y, z)\right]
$$

In this way, the second members of (6) are functions of $X, y, z$ :

$$
\begin{aligned}
& Y=Y(X ; y, z) \\
& Z=Z(X ; y, z)
\end{aligned}
$$

The parameters $y, z$ may be regarded as Gaussian coördinates on $\Sigma$, and the equations (7) define, then, for fixed $y, z$, the curve of $\mathfrak{F}$ which is orthogonal to $\Sigma$ at the point of coorrdinates $y, z$-and when $y$ and $z$ are variable, the congruence of curves in $\mathfrak{F}$ determined by orthogonality to $\Sigma$. 
Adjoining to (7) the equations obtained by differentiation with respect to $X$, and also the identity $X=X$, we have in the five equations

$X=X, Y=Y(X, y, z), \quad Z=Z(X, y, z), Y^{\prime}=Y^{\prime}(X, y, z), Z^{\prime}=Z^{\prime}(X, y, z)$,

a representation of the field of lineal elements determined by the congruence in terms of $X, y, z$ as parameters. The condition (5) that the congruence be normal here takes the form

$$
\begin{gathered}
\left(Y^{\prime} G-Z^{\prime} F\right)(Y Z)+\left(1+Z^{\prime 2}\right)\left(Y Y^{\prime}\right)-Y^{\prime} Z^{\prime}\left(Y Z^{\prime}\right)-Y^{\prime} Z^{\prime}\left(Z Y^{\prime}\right) \\
-\left(1+Y^{\prime 2}\right)\left(Z^{\prime} Z\right)=0,
\end{gathered}
$$

where the parentheses such as $(Y Z)$ represent determinants in the matrix

$$
\left|\begin{array}{cccc}
Y_{y} & Z_{y} & Y_{y}^{\prime} & Z_{y}^{\prime} \\
Y_{z} & Z_{z} & Y_{z}^{\prime} & Z_{z}^{\prime}
\end{array}\right| .
$$

We introduce the notation

$$
\begin{array}{lll}
\omega_{1}=(Y Z), & \omega_{2}=\left(Y Y^{\prime}\right), & \omega_{3}=\left(Y Z^{\prime}\right), \\
\omega_{4}=\left(Z Y^{\prime}\right), & \omega_{5}=(Z Z), & \omega_{6}=\left(Y^{\prime} Z^{\prime}\right),
\end{array}
$$

by means of which (8) abbreviates to

(9) $\left(Y^{\prime} G-Z^{\prime} F\right) \omega_{1}+\left(1+Z^{\prime 2}\right) \omega_{2}-Y^{\prime} Z^{\prime} \omega_{3}-Y^{\prime} Z^{\prime} \omega_{4}-\left(1+Y^{\prime 2}\right) \omega_{5}=0$.

In all that follows we think of $y$ and $z$ as having fixed though arbitrary values, determining thus a single but arbitrary curve $C$ of the congruence orthogonal to $\Sigma$. All the functions of the analysis become in this way functions of the one variable $X$, the abscissa along $C$.

4. The Linear differential equations of the $\omega$ 'S

The next step is to show how the six w's may be uniquely defined by a certain system of linear differential equations together with certain initial conditions. 
We have under consideration a congruence of curves

$$
\begin{aligned}
& Y=Y(X ; y, z), \\
& Z=Z(X ; y, z)
\end{aligned}
$$

selected from the totality of curves obeying the differential equations

$$
\begin{aligned}
& Y^{\prime \prime}=F\left(X, Y, Z, Y^{\prime}, Z^{\prime}\right), \\
& Z^{\prime \prime}=G\left(X, Y, Z, Y^{\prime}, Z^{\prime}\right)
\end{aligned}
$$

It follows by $\S 1$ that the parametric derivatives $Y_{y}, Z_{y}$ obey the linear differential equations

$$
\left(Y_{\bar{y}}\right)^{\prime \prime}=F_{y}\left(Y_{y}\right)+F_{z}\left(Z_{y}\right)+F_{y^{\prime}}\left(Y_{y}\right)^{\prime}+F_{z^{\prime}}\left(Z_{y}\right)^{\prime}
$$

$$
\left(Z_{y}\right)^{\prime \prime}=G_{y}\left(Y_{y}\right)+G_{z}\left(Z_{y}\right)+G_{y^{\prime}}\left(Y_{y}\right)^{\prime}+G_{z^{\prime}}\left(Z_{y}\right)^{\prime}, *
$$

and that $Y_{z}, Z_{z}$ obey the same differential equations:

$$
\left(Y_{z}\right)^{\prime \prime}=F_{y}\left(Y_{z}\right)+F_{z}\left(Z_{z}\right)+F_{y^{\prime}}\left(Y_{z}\right)^{\prime}+F_{z}\left(Z_{z}\right)^{\prime}
$$

$$
\left(Z_{z}\right)^{\prime \prime}=G_{y}\left(Y_{z}\right)+G_{z}\left(Z_{z}\right)+G_{y^{\prime}}\left(Y_{z}\right)^{\prime}+G_{z^{\prime}}\left(Z_{z}\right)^{\prime}
$$

From $\left(10_{1}\right)$ and $\left(10_{2}\right)$ the deduction is direct that the six $\omega$ 's obey a system of linear equations of the first order. Consider, for example,

$$
\omega_{z}=\left|\begin{array}{ll}
Y_{y} & Y_{y}^{\prime} \\
Y_{z} & Y_{z}^{\prime}
\end{array}\right|,
$$

and differentiate it with respect to $X$ :

$$
\omega_{2}^{\prime}=\left|\begin{array}{cc}
Y_{y} & Y_{y}^{\prime \prime} \\
Y_{z} & Y_{z}^{\prime \prime}
\end{array}\right| .
$$

* For convenience of writing, we use small letters as subscripts instead of capitals in denoting the partial derivatives of $F$ and $G$. 
When in the last determinant we substitute for $Y_{y}^{\prime \prime}, Y_{z}^{\prime \prime}$ by the equations $\left(10_{1}\right)$, $\left(10_{\mathbf{z}}\right)$, we find

that is,

$$
\omega_{2}^{\prime}=F_{z}\left|\begin{array}{cc}
Y_{y} & Z_{y} \\
Y_{z} & Z_{z}
\end{array}\right|+F_{y^{\prime}}\left|\begin{array}{cc}
Y_{y} & Y_{y}^{\prime} \\
Y_{z} & Y_{z}^{\prime}
\end{array}\right|+F_{z^{\prime}}\left|\begin{array}{cc}
Y_{y} & Z_{y}^{\prime} \\
Y_{z} & Z_{z}^{\prime}
\end{array}\right|,
$$

$$
\omega_{2}^{\prime}=F_{z} \omega_{1}+F_{y^{\prime}} \omega_{2}+F_{z^{\prime}} \omega_{3} \text {. }
$$

In similar manner the derivative of each $\omega$ is expressible as a linear combination of $\omega$ 's, giving the system of equations

$$
\begin{aligned}
& \omega_{1}^{\prime}=\omega_{3}-\omega_{4}, \\
& \omega_{2}^{\prime}=F_{z} \omega_{1}+F_{y^{\prime}} \omega_{2}+F_{z^{\prime}} \omega_{3}, \\
& \omega_{3}^{\prime}=G_{z} \omega_{1}+G_{y^{\prime}} \omega_{2}+G_{z^{\prime}} \omega_{3}+\omega_{0} \text {, } \\
& \omega_{4}^{\prime}=-F_{y} \omega_{1}+F_{y^{\prime}} \omega_{4}-F_{z^{\prime}} \omega_{5}-\omega_{6} \text {, } \\
& \omega_{5}^{\prime}=\begin{array}{ll}
G_{y} \omega_{1} & -G_{y^{\prime}} \omega_{4}+G_{z^{\prime}} \omega_{5},
\end{array} \\
& \omega_{6}^{\prime}=\quad-G_{y} \omega_{2}+F_{y} \omega_{3}-G_{z} \omega_{4}-F_{z} \omega_{5}+\left(F_{y^{\prime}}+G_{z^{\prime}}\right) \omega_{6} .
\end{aligned}
$$

The $\omega$ 's, as solutions of a system of differential equations of the first order, will be completely determined if we know their values for an initial value of $X$. To find these, we use the power series expansions (6). of the equations of $C$,

$$
\begin{aligned}
& Y=y+p(X-x)+\frac{1}{2} F(X-x)^{2}+\cdots, \\
& Z=z+q(X-x)+\frac{1}{2} G(X-x)^{2}+\cdots
\end{aligned}
$$

We recall that $x$ and the coefficients $p, q, F, G, \ldots$ are functions of $y$ and $z$, coordinates on the surface

$$
\Sigma: x=-\varphi(y, z),
$$

and that $p, q ; r, s, t$ are used to represent the partial derivatives of the first and second orders of the function $\varphi$. With this in mind, we find, on differentiating the equations (7) with respect to $y$,

$$
\begin{aligned}
& Y_{y}=\overline{1+p^{2}}+\overline{r+p F}(X-x)+\cdots, \\
& Z_{y}=p q+\overline{s+p G}(X-x)+\cdots .
\end{aligned}
$$


Therefore, for $X=x$-marking the point of intersection of $C$ with $\mathbf{\Sigma}$

$$
Y_{y}=1+p^{2}, \quad Z_{y}=p q, \quad Y_{y}^{\prime}=r+p F, \quad Z_{y}^{\prime}=s+p G .
$$

Similarly, for $X=x$,

$$
Y_{z}=p q, \quad Z_{z}=1+q^{2}, \quad Z_{y}^{\prime}=s+q F, \quad Z_{z}^{\prime}=t+q G .
$$

Hence the values of the w's for $X=x$ are

$$
\begin{aligned}
& \alpha_{1}=1+p^{2}+q^{2} \\
& \alpha_{2}=q F-p q r \quad+\left(1+p^{2}\right) s, \\
& \alpha_{3}=q G \quad-p q s \quad+\left(1+p^{2}\right) t, \\
& \alpha_{4}=-p F-\left(1+q^{2}\right) r+p q s \text {, } \\
& \alpha_{5}=p G \quad+\left(1+q^{2}\right) s \quad-p q t, \\
& \alpha_{6}=\quad q G r-(q F+p G) s+p F t+\left(r t-s^{2}\right) \text {. }
\end{aligned}
$$

5. The infinite system of Monge-Ampère EQUATIONS

The essential elements in the present analysis are three in number:

(i) the system of linear differential equations (11);

(ii) the initial 'values $\alpha$ of the $\omega$ 's;

(iii) the linear relation (9).

The necessary and sufficient condition that the congruence of curves selected from the quadruply infinite family $\mathfrak{F}$ by the condition of orthogonality to $\mathbf{\Sigma}$ be a normal congruence is that the six functions $\omega$ that obey the differential equations (11), and take for $X=x$ the values $\alpha$, shall satisfy the linear relation (9).

In order to obtain conciseness and symmetry of notation, we condense the equations (11) to

$$
\begin{gathered}
\omega_{1}^{\prime}=p_{11} \omega_{1}+\cdots+p_{16} \omega_{6}, \\
\cdot \cdot \cdot \cdot \cdot \cdot \cdot \cdot \cdot \cdot \cdot \\
\omega_{6}^{\prime}=p_{61} \omega_{1}+\cdots+p_{66} \omega_{6},
\end{gathered}
$$

and rewrite the linear condition (9) as

$$
A_{1} \omega_{1}+\cdots+A_{6} \omega_{6}=0,
$$

using, that is, the abbreviations

$$
\begin{array}{lll}
A_{1}=Y^{\prime} G-Z^{\prime} F, & A_{2}=1+Z^{\prime 2}, & A_{3}=-Y^{\prime} Z^{\prime} \\
A_{4}=-Y^{\prime} Z^{\prime}, & A_{5}=-\left(1+Y^{\prime 2}\right), & A_{6}=0
\end{array}
$$


Our requirement is that the relation (14) subsist identically in $X$ when the $\omega$ 's have the values defined by the differential equations (13) together with the initial values (12). A necessary and sufficient condition that (14) subsist identically in $X$ is that the function of $X$

$$
\Omega \equiv A_{1} \omega_{1}+\cdots+A_{6} \omega_{6},
$$

together with its derivatives of all orders, vanish for $X=x$.

All these derivatives are, like $\Omega$ itself, linear expressions in the $\omega$ 's. In fact, when in the first derivative of $\Omega$,

$$
\Omega^{\prime} \equiv A_{1} \omega_{1}^{\prime}+\cdots+A_{6} \omega_{6}^{\prime}+A_{1}^{\prime} \omega_{1}+\cdots+A_{6}^{\prime} \omega_{6},
$$

we substitute for the $\omega^{\prime}$ 's by (11), we find

where

$$
\boldsymbol{\Omega}^{\prime} \equiv B_{1} \omega_{1}+\cdots+B_{6} \omega_{6},
$$

$$
B_{i}=A_{i}^{\prime}+\sum_{j=1}^{j=6} p_{j i} A_{j}
$$

By repeated differentiation and use of (11) we obtain the infinite sequence

$$
\begin{aligned}
\Omega & \equiv A_{1} \omega_{1}+\cdots+A_{6} \omega_{6}, \\
\Omega^{\prime} & \equiv B_{1} \omega_{1}+\cdots+B_{6} \omega_{6}, \\
\Omega^{\prime \prime} & \equiv C_{1} \omega_{1}+\cdots+C_{6} \omega_{6}, \\
\Omega^{\prime \prime \prime} & \equiv D_{1} \omega_{1}+\cdots+D_{6} \omega_{6},
\end{aligned}
$$

where the coefficients in each linear expression are derived from those in the preceding by the same formulas that give the $B$ 's in terms of the $A$ 's:

$$
\begin{aligned}
C_{i} & =B_{i}^{\prime}+\sum_{j=1}^{j=6} p_{j i} B_{j}, \\
D_{i} & =C_{i}^{\prime}+\sum_{j=1}^{j=6} p_{j i} C_{j},
\end{aligned}
$$


In demanding that

$$
\boldsymbol{\Omega}, \boldsymbol{\Omega}^{\prime}, \boldsymbol{\Omega}^{\prime \prime}, \ldots
$$

all vanish for $X=x$, we get the infinite system of equations

$$
\begin{aligned}
& A_{1} \alpha_{1}+\cdots+A_{6} \alpha_{6}=0 \\
& B_{1} \alpha_{1}+\cdots+B_{6} \alpha_{6}=0 \\
& C_{1} \alpha_{1}+\cdots+C_{6} \alpha_{6}=0
\end{aligned}
$$

where the $A, B, C, \ldots$ of these equations are the values for $X=x$ of the same letters in (17).

The $A, B, C, \ldots$ of (17) are functions of $X, Y, Z, Y^{\prime}, Z^{\prime}$, these being relative to a point moving along the curve $C$. The value $X=x$ corresponds to the intersection of $C$ with $\Sigma$, where we have

$$
X=x, \quad Y=y, \quad Z=z, \quad Y^{\prime}=p, \quad Z^{\prime}=q
$$

Thus the $A, B, C, \ldots$ of (18) are derived from the $A, B, C, \ldots$ of (17) by the substitution (19)-they are functions of $x, y, z, p, q$, coördinates and first partial derivatives relative to $\Sigma$. In (18) and hereafter we shall assume the substitution (19) to be sufficiently well indicated by the context, without employing for it any special notation.

The accent in the formulas (16) denotes total differentiation with respect to $X$, so that applied to any function of $X, Y, Z, Y^{\prime}, Z^{\prime}$, it is equivalent to the operator

$$
\frac{\partial}{\partial X}+Y^{\prime} \frac{\partial}{\partial Y}+Z^{\prime} \frac{\partial}{\partial Z}+Y^{\prime \prime} \frac{\partial}{\partial Y^{\prime}}+Z^{\prime \prime} \frac{\partial}{\partial Z^{\prime}},
$$

or, after the use of the differential equations (1) of $C$ and the substitution (19), to the operator

$$
\frac{\partial}{\partial x}+p \frac{\partial}{\partial y}+q \frac{\partial}{\partial z}+F \frac{\partial}{\partial p}+G \frac{\partial}{\partial q}
$$

Rewritten in accordance with (19), the values (15) of the letters $A$ become

$$
\begin{array}{lll}
A_{1}=p G-q F, & A_{2}=1+q^{2}, & A_{3}=-p q, \\
A_{4}=-p q, & A_{6}=-\left(1+p^{2}\right), & A_{6}=0 .
\end{array}
$$


The recurrence formulas (16) are, in expanded form,

$$
\begin{array}{lll}
B_{1}=A_{1}^{\prime} & +F_{z} A_{2}+G_{z} A_{3}-F_{y} A_{4}+G_{y} A_{5}, & \\
B_{2}=A_{2}^{\prime} \quad+F_{p} A_{2}+G_{p} A_{3} & -G_{y} A_{6}, \\
B_{3}=A_{3}^{\prime}+A_{1}+F_{q} A_{2}+G_{q} A_{8} & +F_{y} A_{6}, \\
B_{4}=A_{4}^{\prime}-A_{1} & +F_{p} A_{4}-G_{p} A_{5}-G_{z} A_{6}, \\
B_{5}=A_{5}^{\prime} & -F_{q} A_{4}+G_{q} A_{5}-F_{z} A_{6}, \\
B_{6}=A_{6}^{\prime} & +A_{3}-A_{4} .
\end{array}
$$

These furnish a definite mechanism whereby the values of the symbols $A$, $B, C, \ldots$ may be successively determined. These symbols are seen to be expressions of higher and higher order in the partial derivatives of $F$ and $G$.

We shall calculate, in addition to the $A$ 's, the explicit values of the $B$ 's only, which are

$$
\begin{aligned}
& B_{1}=p G_{x}-q F_{x}-G_{y}+F_{z}+p\left(F G_{p}+G G_{q}\right)-q\left(F F_{p}+G F_{q}\right) \\
& B_{2}=2 q G+\left(1+q^{2}\right) F_{p}-p q G_{p} \\
& B_{3}=-2 q F+\left(1+q^{2}\right) F_{q}-p q G_{q} \\
& B_{4}=-2 p G-p q F_{p}+\left(1+p^{2}\right) G_{p} \\
& B_{6}=-2 p F+p q F_{q}-\left(1+p^{2}\right) G_{q} \\
& B_{6}=0
\end{aligned}
$$

On substituting the values (12) of the $\alpha$ 's in (18), we obtain the following infinite system of partial differential equations of the Monge-Ampere type:

$$
\begin{aligned}
& \mathfrak{A}_{0}\left(r t-s^{2}\right)+\mathfrak{A}_{1} r+\mathfrak{A}_{2} s+\mathfrak{A}_{3} t+\mathfrak{A}_{4}=0, \\
& \mathfrak{B}_{0}\left(r t-s^{2}\right)+\mathfrak{B}_{1} r+\mathfrak{B}_{2} s+\mathfrak{B}_{3} t+\mathfrak{B}_{4}=0, \\
& \mathfrak{S}_{0}\left(r t-s^{2}\right)+\mathfrak{C}_{1} r+\mathfrak{C}_{2} s+\mathfrak{C}_{3} t+\mathfrak{C}_{4}=0,
\end{aligned}
$$


where

$$
\begin{aligned}
\mathfrak{A}_{0} & =A_{6}, \\
\mathfrak{A}_{1} & =-p q A_{2}-\left(1+q^{2}\right) A_{4}+q G A_{6}, \\
(24) \mathfrak{A}_{2} & =\left(1+p^{2}\right) A_{2}-p q A_{3}+p q A_{4}+\left(1+q^{2}\right) A_{5}-(q F+p G) A_{6}, \\
\mathfrak{U}_{3} & =\left(1+p^{2}\right) A_{3}-p q A_{5}+p F A_{6}, \\
\mathfrak{U}_{4} & =\left(1+p^{2}+q^{2}\right) A_{1}+q F A_{2}+q G A_{3}-p F A_{4}+p G A_{5},
\end{aligned}
$$

and the values of $\mathfrak{B}, \mathfrak{C}, \ldots$ are derived respectively from those of $B, C, \ldots$ by the same formulas.

A surface gives rise to a normal congruence when and only when it satisfies the infinite system (23).

The important cases are those where there exists at least one solution of (23) corresponding to arbitrary initial values of $x, y, z, p, q$, that is, where the system (23) is completely integrable. It is easy to see that otherwise any normal congruences present in our four-fold infinitude of curves are either only finite in number, or else confined to a triply infinite sub-family. The situation in the latter case is as described in the sixth of the first group of results collected in the introduction.

6. Classification. The infinite matrix. Quasi-natural familifs

The classification of quadruply infinite families of curves with respect to the normal congruences contained within them depends on the rank of the infinite matrix

$$
\left|\begin{array}{ccc}
A_{1} & \cdots & A_{6} \\
B_{1} & \cdots & B_{6} \\
C_{1} & \cdots & C_{6} \\
\cdots & \cdots
\end{array}\right|
$$

together with the complete integrability of the system formed by a certain finite number of the equations (23).

For instance, suppose that the $D$ row of the matrix is a linear combination of the preceding rows:

$$
D_{i}=\varrho A_{i}+\sigma B_{i}+\tau C_{i}
$$


Then it is easily shown by the recurrence formulas (16) that every subsequent row must be a linear combination of its preceding rows; in other words, for the matrix to be of rank three it is necessary and sufficient that

$$
|A B C D|=0, \quad|A B C| \neq 0,
$$

where in writing a matrix equal to zero we mean that every determinant resulting from it by the suppression of columns is equal to zero.

The subsistence of the first three equations of (23) will then imply that of all the succeeding ones. The problem of the complete integrability of (23) is thus referred to that of its first three equations, a matter dealt with in the standard theory.*

It is to be observed that

$$
\mathfrak{A}_{0}\left(r t-s^{2}\right)+\mathfrak{A}_{1} r+\mathfrak{A}_{2} s+\mathfrak{A}_{3} t+\mathfrak{A}_{4}=0
$$

is in reality not present, for by (24) and (20) it disappears identically. This results from the fact that the congruence is constructed orthogonal to $\Sigma$. In the case of rank $r$, therefore, all and only the surfaces obeying the $r-1$ equations $\left(23_{z}\right)$ to $\left(23_{r}\right)$ give normal congruences.

In particular, when

$$
|A B|=0
$$

$(r=1)$, an arbitrary surface will produce a normal congruence, and conversely. The condition (25) furnishes by (20), (22) a system of partial differential equations for $F$ and $G$, whose solution gives for those functions the forms (3), characteristic of natural families. We thus have the theorem of Thomson and Tait together with the converse theorem of Kasner stated in the introduction.

The most interesting new case is the next one, of rank two,

$$
|A B C|=0, \quad|A B| \neq 0 .
$$

The families defined by these conditions we term quasi-natural.

Their characteristic property is that every surface satisfying the equation $\left(23_{2}\right)$ (which actually is linear, since in fact $\mathfrak{B}_{0}=0$ ) determines a normal

\footnotetext{
* E. Goursat, Leçons sur l'Intégration des Équations aux Dérivées partielles du second Ordre, tome II, chapter VI.
} 
congruence, while the same is not true of an arbitrary surface. Since the subsistence of $\left(23_{g}\right)$ means that the congruence orthogonal to the surface $\Sigma$ has an orthogonal surface consecutive to $\Sigma$, a quasi-natural family may be described as a non-natural family in which every congruence admitting two consecutive orthogonal surfaces is normal.

It is evidently significant to inquire as to consistency of the conditions (26). The following example, establishing the existence of quasi-natural families, may be of interest.

Suppose that a family of $\infty^{4}$ curves in space has these two properties:

(1) its curves are vertical plane curves;

(2) it possesses cylindrical symmetry with respect to every vertical line; that is, every curve of the family remains in the family when rotated about any vertical axis.

For instance, the $\infty^{4}$ vertical circles of a given radius.

By solving a simple functional equation, it can be shown that such families are characterized by differential equations of the form

$$
\begin{aligned}
& y^{\prime \prime}={y^{\prime}}^{2} f\left(\frac{1+{z^{\prime}}^{2}}{y^{\prime 2}}, y\right) \\
& z^{\prime \prime}=0
\end{aligned}
$$

(the axis of $y$ being assumed vertical). Provided that

$$
f \neq\left(\frac{1+{z^{\prime}}^{2}}{y^{\prime 2}}+1\right) f_{1}(y),
$$

the family is not natural.

Consider an arbitrary vertical plane $\boldsymbol{I}$, together with an arbitrary curve $\boldsymbol{r}$ in the plane. II contains $\infty^{2}$ curves of the family, of which $\infty^{1}$ meet $\Gamma$ at right angles. Imagine $\boldsymbol{I}$ to be rolled on a vertical cylinder of arbitrary cross section. Then $\Gamma$ generates a moulure surface $M$. The rolling is equivalent to a succession of infinitesimal rotations about the vertical elements of the

\footnotetext{
* The sub-case where $f$ does not involve $y$ occurs in Professor Kasner's paper (p. 136), being arrived at from an entirely different point of view. The statement on page 136 to the effect that not all curve systems of the form $y^{\prime \prime}=y^{\prime 2} f\left(\left(1+z^{\prime 2}\right) / y^{\prime 2}\right), z^{\prime \prime}=0$ have the property that an arbitrary point taken as base determines a normal congruence, is an error, as follows from the fact that the moulure surface $M$ which we describe below may be allowed to degenerate to an arbitrary point. The Pfaffian on page 137, loc. cit., has an integrating factor.
} 
cylinder. Evidently, therefore, the $\infty^{1}$ curves of the family meeting $\Gamma$ orthogonally in its plane generate $\infty^{2}$ curves which belong to the family and are orthogonal to $M$.

Now, the $\infty^{1}$ curves in $\Pi$ that are orthogonal to $\Gamma$ admit $\infty^{1}$ orthogonal trajectories. In the rolling of $\boldsymbol{I}$ these generate $\infty^{1}$ moulure surfaces at right angles to the $\infty^{2}$ curves orthogonal to $M$; in other words, these $\infty^{2}$ curves are a normal congruence.

It is only necessary to remark that the vertical moulure surfaces of space are defined by the linear partial differential equation of the second order

$$
\left(1+q^{2}\right) s-p q t=0
$$

to complete the characterization of the families defined by (27), (28) as quasi-natural.

We omit the details of the study of the higher ranks of the infinite matrix. The results are summarized in the introduction. We have found examples to demonstrate the effective existence of each of the types there described.

\section{Part II}

\section{THE CONGRUENCES BASED ON ARBITRARY POINTS, PLANES, SPHERES, AND STRAIGHT LINES}

7. THE GENERAL PRoblem

In this part of the paper, the characteristic feature of the analysis is that the initial values $\alpha$ in the formulation of $\S 5$ are functions of a parameter instead of constants.

In the family $\mathfrak{F}$ defined by the differential equations

$$
\begin{aligned}
& y^{\prime \prime}=F\left(x, y, z, y^{\prime}, z^{\prime}\right), \\
& z^{\prime \prime}=G\left(x, y, z, y^{\prime}, z^{\prime}\right),
\end{aligned}
$$

the curve which passes through the point $O(x, y, z)$ in the direction $1: p: q$ is defined by the equations

$$
\begin{aligned}
& Y=y+p(X-x)+\frac{1}{2} F(X-x)^{2}+\cdots, \\
& Z=z+q(X-x)+\frac{1}{2} G(X-x)^{2}+\cdots
\end{aligned}
$$


If we regard $x, y, z$ as fixed, while $p, q$ vary, these equations represent the congruence of curves of $\mathfrak{F}$ which pass through $O$, as dependent on the parameters $p, q$ :

$$
\begin{aligned}
& Y=Y(X ; p, q), \\
& Z=Z(X ; p, q) .
\end{aligned}
$$

The $\omega$ 's in this case are the two-rowed determinants in the matrix

$$
\left|\begin{array}{cccc}
Y_{p} & Z_{p} & Y_{p}^{\prime} & Z_{p}^{\prime} \\
Y_{q} & Z_{q} & Y_{q}^{\prime} & Z_{q}^{\prime}
\end{array}\right|
$$

and are found by (41) to have for $X=x$, that is, at the fixed point $O$, the values

$$
\alpha_{1}=0, \quad \alpha_{2}=0, \quad \alpha_{8}=0, \quad \alpha_{4}=0, \quad \alpha_{5}=0, \quad \alpha_{6}=1
$$

We think of $p, q$ as having arbitrary but fixed values, determining thus a definite curve $C$, which is any curve of the congruence based on $O$. Since the curves passing through an arbitrary point of space are to form a normal congruence, each point of $C$ must serve as the base point of such a congruence. Hence the $x$ for which the $\omega$ 's take the values (30) is a parameter, capable of continuous variation, and the $\alpha$ 's in (30) are therefore to be considered as functions of $x$ having the values (30) for all values of $x$.

The general problem formulated at the beginning of $\S 5$ is therefore to be modified as follows.

We have

(i) a system of linear differential equations of the first order (independent variable denoted by $x$ ),

$$
\omega_{i}^{\prime}=\sum_{j} p_{i j} \omega_{j} \quad(i, j=1,2, \ldots, n)
$$

(ii) a system of $n$ functions of a parameter $x_{0}$,

$$
\omega_{1}^{0}=\alpha_{1}\left(x_{0}\right), \omega_{2}^{0}=\alpha_{2}\left(x_{0}\right), \ldots, \omega_{n}^{0}=\alpha_{n}\left(x_{0}\right) ;
$$

(iii) a linear relation

$$
A_{1} \omega_{1}+A_{2} \omega_{2}+\cdots+A_{n} \omega_{n}=0
$$

whose coefficients are given functions of $x$. 
Our problem is to find the conditions under which the functions $\omega$ that satisfy the equations (i), and take for $x=x_{0}$ the values (ii), obey, for all values of $x_{0}$, the linear relation (iii).

We proceed to obtain necessary conditions.

Suppose that the integral curve (in $(n+1)$-space) of the equations (i) which is determined by the point $x_{0}$ on the curve of initial points (ii) obeys the relation

$$
A_{1}(x) \omega_{1}(x)+\cdots+A_{n}(x) \omega_{n}(x)=0 .
$$

By differentiation and use of (i) it follows that it must also obey the relation

$$
B_{1}(x) \omega_{1}(x)+\cdots+B_{n}(x) \omega_{n}(x)=0,
$$

where the $B$ 's are derived from the $A$ 's by the formula (16 $)$ (with the number 6 replaced by $n$ ).

Repeating this process indefinitely, we are led to the infinite sequence of equations

(31) $\sum A_{i}(x) \omega_{i}(x)=0, \sum B_{i}(x) \omega_{i}(x)=0, \quad \sum C_{i}(x) \omega_{i}(x)=0, \ldots$,

where the successive coefficients are derived by the recurrence formulas (16).

The relations (31) are to be identities in $x$, and must therefore hold in particular for $x=x_{0}$ :

$$
\begin{gathered}
\sum A_{i}\left(x_{0}\right) \alpha_{i}\left(x_{0}\right)=0, \quad \sum B_{i}\left(x_{0}\right) \alpha_{i}\left(x_{0}\right)=0, \\
\sum C_{i}\left(x_{0}\right) \alpha_{i}\left(x_{0}\right)=0, \ldots .
\end{gathered}
$$

In our writing $x=x_{0}$, the letters in (31) change from functions of $x$ to constants, but then become in (32) functions of $x_{0}$ through the circumstance that $x_{0}$ is capable of taking any value. The equations (32) must be identities in $x_{0}$, which makes it permissible, as will be done, to differentiate them any number of times.

It is to be seen that in (32) we have sufficient as well as necessary conditions for the subsistence of (iii) under the conditions (i) and (ii). For (32) expresses that the derivatives of all orders of the first member of (iii) vanish for $x=x_{0}$; this must cause the first member of (iii) to be identically zero in $x$. 
We have therefore to undertake the study of the equations (32)-an infinite system of linear equations in the $\alpha$ 's. As in Part I, all depends on the rank of the matrix

$$
\left|\begin{array}{ccc}
A_{1} & \ldots & A_{n} \\
B_{1} & \cdots & B_{n} \\
C_{1} & \ldots & C_{n} \\
\ldots & \ldots & \cdots
\end{array}\right|
$$

It will prevent circumlocution to say that every determinant of higher than the $n$th order in this matrix is equal to zero.

In the series of sub-matrices of (33),

let the one of $k$ rows

$$
|A|, \quad|A B|, \quad|A B C|, \ldots,
$$

$$
|A B \cdots K|
$$

be the first which is equal to zero (in which every determinant resulting from the suppression of columns is equal to zero):

$$
|A B \cdots K|=0, \quad|A B \ldots J| \neq 0 .
$$

According to the agreement just made, this must happen for $k=n+1$, if not before.

Under (34), the letters $K$ will be linear combinations of $A, B, \ldots, J$ :

$$
K_{i}=\varrho_{1} A_{i}+\varrho_{2} B_{i}+\cdots+\varrho_{k-1} J_{i}
$$

where the $\varrho$ 's are functions of $x_{0}$. Then by the recurrence formulas (16), the next letters $L$ are linearly composable of those preceding them:

$$
L_{i}=\varrho_{1}^{\prime} A_{i}+\left(\varrho_{2}^{\prime}+\varrho_{1}\right) B_{1}+\cdots+\left(\varrho_{k-1}^{\prime}+\varrho_{k-2}\right) J_{i}+\varrho_{k-1} K_{i}
$$

and the same is seen to be true of every row in (33) from the $K$ row on. This is to say that the first $k-1$ equations of (32),

$$
\sum A_{i} \alpha_{i}=0, \quad \sum B^{i} \alpha_{i}=0, \ldots, \quad \sum J_{i} \alpha_{i}=0,
$$

will imply. all the subsequent ones. 
In this way our necessary and sufficient condition-the subsistence of the infinite system (32)-resolves itself into a disjunction of conditions as follows:

In order that the w's defined by (i) and (ii) satisfy (iii), it is necessary and sufficient that one or another of the following $n+1$ mutually exclusive condetions be verified:

$(k)$

$$
\begin{aligned}
& |A|=0 \\
& |A B|=0, \quad|A| \neq 0, \\
& \sum A_{i} \alpha_{i}=0 \\
& |A B C|=0, \quad|A B| \neq 0, \\
& \sum A_{i} \alpha_{i}=0, \quad \sum B_{i} \alpha_{i}=0 \\
& |A B \cdots K|=0, \quad|A B \cdots J| \neq 0, \\
& \sum A_{i} \alpha_{i}=0, \ldots, \sum J_{i} \alpha_{i}=0 ;
\end{aligned}
$$

and so on to

$$
\begin{gathered}
|A B \cdots N| \neq 0, \\
\sum A_{i} \alpha_{i}=0, \ldots, \quad \sum N_{i} \alpha_{i}=0 .
\end{gathered}
$$

This is one form of complete solution of the problem formulated at the beginning of this section. But the problem presents a dual aspect.

Subtract from each of the equations (32) the derivative of the preceding. There results the new system

$$
\sum A_{i} \beta_{i}=0, \quad \sum B_{i} \beta_{i}=0, \quad \sum C_{i} \beta_{i}=0, \ldots
$$

where

$$
\beta_{i}=-\alpha_{i}^{\prime}+\sum_{j=1}^{j=n} p_{i j} \alpha_{j}
$$

Applying the same procedure to the equations ( $\left(32^{\prime}\right)$, and so on indefinitely, we get the doubly infinite array of equations 


$$
\sum A_{i} \alpha_{i}=0, \quad \sum B_{i} \alpha_{i}=0, \quad \sum C_{i} \alpha_{i}=0, \ldots
$$

$$
\begin{array}{lll}
\sum A_{i} \beta_{i}=0, & \sum B_{i} \beta_{i}=0, & \sum C_{i} \beta_{i}=0, \ldots \\
\sum A_{i} \gamma_{i}=0, & \sum B_{i} \gamma_{i}=0, & \sum C_{i} \gamma_{i}=0, \ldots
\end{array}
$$

where the $\gamma$ 's are derived from the $\beta$ 's, the $\delta$ 's from the $\gamma$ 's, etc., by the same formulas which generate the $\beta$ 's from the $\alpha$ 's.

Now this array of equations may as well be generated by its first column

$$
\sum A_{i} \alpha_{i}=0, \quad \sum A_{i} \beta_{i}=0, \quad \sum A_{i} \gamma_{i}=0, \ldots
$$

as by its first row. For by adding each equation in the first column to the derivative of its predecessor, the second column of equations results, and iteration of this process gives the whole array.

Since the equations (38) thus imply and are implied by the equations (32), they represent, like (32), a necessary and sufficient condition for our problem. And like (32) they are equivalent to a disjunction of mutually exclusive conditions, namely

$$
\begin{aligned}
|\boldsymbol{\alpha} \beta \boldsymbol{\gamma}| & =0, & |\boldsymbol{\alpha} \boldsymbol{\beta}| & \neq 0, \\
\sum A_{i} \alpha_{i} & =0, & \sum A_{i} \beta_{i} & =0 ;
\end{aligned}
$$

and so on to

$$
\begin{gathered}
|\boldsymbol{\alpha} \beta \cdots \nu| \neq 0, \\
\sum A_{i} \alpha_{i}=0, \cdots, \sum A_{i} \nu_{i}=0 .
\end{gathered}
$$

It is easy to prove through (37) that the case $(k)$ of (35) implies the case $(n-k+2)$ of (39) except for its inequality, and vice versa. For this reason we need never use matrices of order greater than $\frac{1}{2}(n+2)$. Moreover, the elements $\alpha, \beta, \gamma, \ldots$ may be much simpler than $A, B, C, \ldots$. These remarks will be of great advantage to us in the applications that follow. 
8. WhEN THE BASE MANIFOLD IS A POINT

In the problem of normal congruences, the recurrence formulas for $\boldsymbol{\beta}, \boldsymbol{\gamma}, \boldsymbol{\delta}, \ldots$ are

$$
\begin{aligned}
& \beta_{1}=-\alpha_{1}^{\prime} \quad+\alpha_{3}-\alpha_{4}, \\
& \beta_{2}=-\alpha_{2}^{\prime}+F_{2} \alpha_{1}+F_{p} \alpha_{2}+F_{q} \alpha_{3}, \\
& \text { (40) } \\
& \beta_{3}=-\alpha_{8}^{\prime}+G_{z} \alpha_{1}+G_{p} \alpha_{2}+G_{q} \alpha_{3}+\alpha_{6}, \\
& \beta_{4}=-\alpha_{4}^{\prime}-F_{y} \alpha_{1} \\
& +F_{p} \alpha_{4}-F_{q} \alpha_{5} \quad-\alpha_{6} \\
& \beta_{5}=-\alpha_{5}^{\prime}+G_{y} \alpha_{1} \\
& -G_{p} \alpha_{4}+G_{q} \alpha_{5} \\
& \beta_{6}=-\alpha_{6}^{\prime} \\
& -G_{y} \alpha_{2}+F_{y} \alpha_{8}-G_{z} \alpha_{4}-F_{z} \alpha_{5}+\left(F_{p}+G_{q}\right) \alpha_{6},
\end{aligned}
$$

the accent denoting the operator $(\partial / \partial x)+p(\partial / \partial y)+q(\partial / \partial z)+F(\partial / \partial p)$ $+G(\partial / \partial q)$. Also, the equation $\sum A_{i} \alpha_{i}=0$ disappears identically, this corresponding to the fact that in every case the congruence starts orthogonally to its base manifold.

Our four problems of the point, plane, sphere, and straight line are each distinguished by a particular form of the functions $\alpha$.

For the point as base manifold, we have (see (30))

(41) $\alpha_{1}=0, \quad \alpha_{2}=0, \quad \alpha_{8}=0, \quad \alpha_{4}=0, \quad \alpha_{5}=0, \quad \alpha_{6}=1$

Calculation gives

$\left(41^{\prime}\right) \beta_{1}=0, \quad \beta_{2}=0, \quad \beta_{3}=1, \quad \beta_{4}=-1, \quad \beta_{5}=0, \quad \beta_{6}=F_{p}+G_{q}$

$$
\begin{array}{r}
\gamma_{1}=2, \quad \gamma_{2}=F_{q}, \quad \gamma_{3}=2 G_{q}+F_{p}, \quad \gamma_{4}=-2 F_{p}-G_{q}, \\
\gamma_{5}=G_{p}, \quad \gamma_{6}=-\left(F_{p}+G_{q}\right)^{\prime}+F_{y}+G_{z}+\left(F_{p}+G_{q}\right)^{2}
\end{array}
$$

$$
\begin{aligned}
& \delta_{1}=3\left(F_{p}+G_{q}\right), \\
& \delta_{2}=-F_{q}+2 F_{z}+2 F_{q}\left(F_{p}+G_{q}\right) \text {, } \\
& \left(41^{\prime \prime \prime}\right) \begin{array}{r}
\delta_{3}=-2 F_{p}^{\prime}-3 G_{q}^{\prime}+F_{y}+3 G_{z}+3 G_{q}\left(F_{p}+G_{q}\right)+F_{p}^{2}+F_{q} G_{p}, \\
\boldsymbol{\delta}_{4}=2 G_{q}^{\prime}+3 F_{p}^{\prime}-G_{z}-3 F_{y}-3 F_{p}\left(F_{p}+G_{q}\right)-G_{q}^{2}-F_{p} G_{q},
\end{array} \\
& \delta_{5}=-G_{p}^{\prime}+2 G_{y}+2 G_{p}\left(F_{p}+G_{q}\right) \text {, } \\
& \delta_{6}=\cdot \cdot \cdot \cdot \cdot \cdot \cdot \cdot \cdot \cdot \cdot
\end{aligned}
$$


It is easily veritied that here, besides $\sum A_{i} \alpha_{i}=0$, we have $\sum B_{i} \alpha_{i}=0$. Hence, by (35), if

or if

$$
|A B|=0
$$

$$
|A B C|=0, \quad|A B| \neq 0,
$$

the congruence determined by an arbitrary point of space will be normal. Thus, in every natural or quasi-natural family the congruence based on an arbitrary point is normal.

We have next to consider

$$
\begin{gathered}
|A B C D|=0, \quad|A B C| \neq 0 \\
\sum A_{i} \alpha_{i}=0, \quad \sum B_{i} \alpha_{i}=0, \quad \sum C_{i} \alpha_{i}=0
\end{gathered}
$$

These equations written in expanded form would be very complicated on account of the complication of the explicit expressions of the $C^{\prime} \mathrm{s}$ and $D$ 's; but by the remarks at the end of the preceding section they imply

$$
|\alpha \beta \gamma \delta|=0 \text {, }
$$

$$
\sum A_{i} \alpha_{i}=0, \quad \sum A_{i} \beta_{i}=0, \quad \sum A_{i} \gamma_{i}=0 ;
$$

and, conversely, (39)(4') implies (35) (4) except for the condition $|A B C| \neq 0$, since in fact $|\alpha \beta \gamma| \neq 0$ (thus completing the system (39)(4)), indeed

$$
\left(\alpha_{1} \beta_{3} \gamma_{6}\right)=-2 \text {. }
$$

$(39)\left(4^{\prime}\right)$ gives the following system of four partial differential equations for $F$ and $G$ :

$$
\begin{gathered}
F_{q}\left(F_{p}+G_{q}\right)+4 F_{z}-2\left(F_{q x}+p F_{q y}+q F_{q z}+F F_{p q}+G F_{q q}\right)=0 \\
G_{p}\left(F_{p}+G_{q}\right)+4 G_{y}-2\left(G_{p x}+p G_{p y}+q G_{p z}+F G_{p p}+G G_{p q}\right)=0 \\
\left(1+q^{2}\right) F_{q}+p q\left(F_{p}-G_{q}\right)-\left(1+p^{2}\right) G_{p}+2(p G-q F)=0 \\
p G_{x}-q F_{x}-G_{y}+F_{z}=0
\end{gathered}
$$

* By this notation we denote (39) (4) without its inequality, $|\alpha \beta \gamma| \neq 0$. 
from the solutions of which we are to exclude those of $|A B C|=0$. The existence of a solution of (43) which is not simultaneously one of $|A B C|=0$, that is, not natural or quasi-natural, is proved by the example

$$
y^{\prime \prime}=y^{\prime 2}, \quad z^{\prime \prime}=z^{\prime 2} .
$$

There are no further possibilities here than those already enumerated. For the next case is

$$
\begin{gathered}
|A B C D E|=0, \quad|A B C D| \neq 0, \\
\sum A_{i} \alpha_{i}=0, \ldots, \sum D_{i} \alpha_{i}=0 .
\end{gathered}
$$

But these conditions imply (39)(3), which includes

whereas

$$
|\alpha \beta \gamma|=0
$$

$$
\left(\alpha_{1} \beta_{3} \gamma_{6}\right)=-2 \neq 0 \text {. }
$$

9. When the BaSE MANIFOLD IS A PLANE

We write the equation of the plane in the form

$$
x=-\varphi(y, z)=-(a y+b z+c) .
$$

I'he $\alpha$ 's are given by (12), where we write $r=s=t=0$, and find

$$
\begin{aligned}
\alpha_{1}=1+p^{2}+q^{2}, \quad \alpha_{2}=q F, \quad \alpha_{3}=q G, \\
\alpha_{4}=-p F, \quad \alpha_{5}=p G, \quad \alpha_{6}=0 .
\end{aligned}
$$

By applying the formulas (40) the $\beta$ 's are found to have the values

$$
\begin{aligned}
& \beta_{1}=-p F-q G, \\
& \beta_{2}=-q F_{x}-p q F_{y}+\left(1+p^{2}\right) F_{z}-F G, \\
& \beta_{3}=-q G_{x}-p q G_{y}+\left(1+p^{2}\right) G_{z}-G^{2}, \\
& \beta_{4}=p F_{x}-\left(1+q^{2}\right) F_{y}+p q F_{z}+F^{\mathbf{z}}, \\
& \beta_{5}=-p G_{x}+\left(1+q^{2}\right) G_{y}-p q G_{z}-F G, \\
& \beta_{6}=G\left(q F_{y}-p F_{z}\right)+F\left(p G_{z}-q G_{y}\right) .
\end{aligned}
$$


We omit the calculation of the higher letters.

Passing over the obvious case of natural families, we must next consider

(35) (3)

$$
\begin{aligned}
|A B C| & =0, & |A B| & \neq 0, \\
\sum A_{i} \alpha_{i} & =0, & \sum B_{i} \alpha_{i} & =0
\end{aligned}
$$

$\sum A_{i} \alpha_{i}=0$ is satisfied identically, in virtue of the orthogonality of the congruence to its base plane, while $\sum B_{i} \alpha_{i}=0$, which by (22) and (45) is in the present case

$$
p G_{x}-q F_{x}-G_{y}+F_{z}=0
$$

can be shown to be a consequence of the conditions

$$
|A B C|=0, \quad|A B| \neq 0 .
$$

For under these conditions, the $C$ 's are linearly composable from the $A$ 's and $B$ 's. Since, by (20), (22), $A_{6}=0, B_{6}=0$, we must have $C_{6}=0$. By the sixth formula (21) (write $B, C$ for $A, B$ ) it follows that $B_{3}=B_{4}$. But, according to (20), $A_{3}=A_{4}$. Therefore, $C_{3}=C_{4}$. This gives, by further reference to (21) (with $B, C$ for $A, B$ ),

$$
2 B_{1}+F_{q} B_{2}+G_{q} B_{8}-F_{p} B_{4}+G_{p} B_{5}+\left(F_{y}+G_{z}\right) B_{6}=0,
$$

which, reduced by (22), is

$$
p G_{x}-q F_{x}-G_{y}+F_{z}=0 .
$$

Therefore, in a quasi-natural family the curves orthogonal to an arbitrary plane form a normal congruence.

There are three further possibilities here, the conditions for which are given respectively by (4), (5), (6) of (35). We have verified the effective existence of the first of these by the example

$$
y^{\prime \prime}=\frac{y^{\prime}\left(1+y^{\prime 2}+z^{\prime 2}\right)}{x+z z^{\prime}}, \quad z^{\prime \prime}=0
$$


described, in another connection, in the introduction, but have not been able to settle the existence question for the other two types.*

If we approach the problem from the dual side of the $\alpha, \beta, \gamma, \ldots$, the first case arrived at is

$$
|\alpha \beta|=0 .
$$

This gives a system of partial differential equations in $F$ and $G$, whose solution is

$$
F=\frac{p\left(1+p^{2}+q^{2}\right) \varphi(\xi)}{\eta \psi(\xi)+\zeta \varphi(\xi)+\varphi(\xi) \psi(\xi)},
$$

$$
G=\frac{q\left(1+p^{2}+q^{2}\right) \psi(\xi)}{\eta \psi(\xi)+\zeta \varphi(\xi)+\varphi(\xi) \psi(\xi)},
$$

where

$$
\xi=x+p y+q z, \quad \eta=x+p y, \quad \zeta=x+q z .
$$

It can be proved that this case is obtainable in the most general way as follows: distribute the $\infty^{8}$ planes of space into $\infty^{2}$ sets of $\infty^{1}$ each, and construct for each set of planes the $\infty^{2}$ orthogonal curves. The type (46) is, in other words, characterized by the property that every plane determines a normal congruence in which the other orthogonal surfaces are also planes.

10. WHEN THE BASE MANIFOLD IS A SPHERE

For a sphere of radius $R$ with its equation in the form $x=-\varphi(y, z)$,

$$
\frac{r}{1+p^{2}}=\frac{s}{p q}=\frac{t}{1+q^{2}}=\frac{\left(1+p^{2}+q^{2}\right)^{1 / 2}}{R} .
$$

Hence, by (12),

$$
\alpha_{1}=1+p^{2}+q^{2},
$$

(47a) $\alpha_{2}=q F$,

$$
\alpha_{8}=q G+\frac{\left(1+p^{2}+q^{2}\right)^{3 / 2}}{R}
$$

*A promising approach is to attempt to determine the arbitrary functions $\varphi$ and $\psi$ in the equations (46) below so as to satisfy the conditions of (35) (5), (35) (6), respectively; but the calculations necessary to carry this through seem toc long. 


$$
\begin{aligned}
& \alpha_{4}=-p F-\frac{\left(1+p^{2}+q^{2}\right)^{3 / 2}}{R}, \\
& \alpha_{5}=p G, \\
& \alpha_{6}=\quad \frac{\left(1+p^{2}+q^{2}\right)^{1 / 2}}{R}(p F+q G)+\frac{\left(1+p^{2}+q^{2}\right)^{2}}{R^{2}} .
\end{aligned}
$$

Using the superscripts $\sigma, \pi, p$ to denote quantities relative to the sphere, plane, and point, respectively, we see by reference to (41), $\left(41^{\prime}\right)$ and (45) that

where

$$
\alpha^{(\sigma)}=\alpha^{(\pi)}+\lambda \alpha^{(p)}+\mu \beta^{(p)},
$$

$\lambda=\frac{\left(1+p^{2}+q^{2}\right)^{1 / 2}}{R}(p F+q G)-\frac{\left(1+p^{2}+q^{2}\right)^{3 / 2}}{R}(p F+q G)+\frac{\left(1+p^{2}+q^{2}\right)^{2}}{R^{2}}$,

$\mu=\frac{\left(1+p^{2}+q^{2}\right)^{2 / 2}}{R}$

that is, the $\alpha$ 's for the sphere are linear combinations of the $\alpha$ 's for the plane and the $\alpha$ 's and $\beta$ 's for the point. On account of the linear homogeneous character of our entire problem, it follows that if arbitrary points and planes give rise to normal congruences, the same will be true of an arbitrary sphere.

Therefore, by the results of the preceding two sections, if the family is natural or quasi-natural, every sphere gives rise to a normal congruence.

We now show that the natural and quasi-natural families are the only ones in which the curves orthogonal to an arbitrary sphere form a normal congruence.

For if the normality property subsists with respect to an arbitrary sphere, it must subsist with respect to arbitrary points and planes; we then have by (37)

$$
\begin{aligned}
& \sum A_{i} \alpha_{i}^{(p)}=0, \quad \sum A_{i} \beta_{i}^{(p)}=0, \quad \sum A_{i} \gamma_{i}^{(p)}=0, \quad \sum A_{i} \alpha_{i}^{(\pi)}=0, \\
& \sum B_{i} \alpha_{i}^{(p)}=0, \quad \sum B_{i} \beta_{i}^{(p)}=0, \quad \sum B_{i} \gamma_{i}^{(p)}=0, \quad \sum B_{i} \alpha_{i}^{(\pi)}=0, \\
& \sum C_{i} \boldsymbol{\alpha}_{i}^{(p)}=0, \quad \sum C_{i} \boldsymbol{\beta}_{i}^{(p)}=0, \quad \sum C_{i} \gamma_{i}^{(p)}=0, \quad \sum C_{i} \boldsymbol{\alpha}_{i}^{(\pi)}=0 \\
& (i=1, \ldots, 6) \text {. }
\end{aligned}
$$

It follows from this system of linear equations that either

or

$$
|A B C|=0
$$

$$
\left|\alpha^{(p)} \beta^{(p)} \gamma^{(p)} \alpha^{(\pi)}\right|=0
$$


Under the first condition the family is natural or quasi-natural.

The second gives, by $(41),\left(41^{\prime}\right),\left(41^{\prime \prime}\right),(45)$, the system of partial differential equations

$$
\begin{aligned}
& \left(1+p^{2}+q^{2}\right) F_{q}-2 q F=0, \\
& \left(1+p^{2}+q^{2}\right) G_{p}-2 p G=0 . \\
& \left(1+p^{2}+q^{2}\right) F_{p}-2 p F=\left(1+p^{2}+q^{2}\right) G_{q}-2 q G,
\end{aligned}
$$

which together with

$$
\sum B_{i} \alpha_{i}^{(\pi)}=0
$$

whose expansion by (22) and (45) is

$$
p G_{x}-q F_{x}-G_{y}+F_{z}=0,
$$

determine for $F$ and $G$ the rorms

$$
\begin{aligned}
& F=\left(L_{y}-p L_{x}\right)\left(1+p^{2}+q^{2}\right), \\
& G=\left(L_{z}-q L_{x}\right)\left(1+p^{2}+q^{2}\right),
\end{aligned}
$$

that are characteristic of natural families.

This completes the proof of the theorem stated above.

11. When the BASE MANIFold IS A STRAight LiNe

Consider the congruence in a system of $\infty^{4}$ curves which is determined by orthogonality to an arbitrary straight line

$$
\begin{aligned}
& y=a x+c, \\
& z=b x+d .
\end{aligned}
$$

If $1: p: q$ is a direction perpendicular to the line,

$$
1+a p+b q=0 .
$$


The last three equations determine $y, z, q$ in terms of $x$ and $p$, so that $x$ and $p$ may be used as the parameters of the congruence. The $\omega$ 's are then the determinants of the matrix

$$
\left|\begin{array}{llll}
Y_{x} & Z_{x} & Y_{x}^{\prime} & Z_{x}^{\prime} \\
Y_{p} & Z_{p} & Y_{p}^{\prime} & Z_{p}^{\prime}
\end{array}\right|
$$

The initial values of the $\omega$ 's are found to be

$$
\alpha_{i}=a^{2} \alpha_{i}^{(1)}+a b \alpha_{i}^{(2)}+b^{2} \alpha_{i}^{(8)}
$$

where

$$
\alpha_{1}^{(1)}=0, \quad \alpha_{2}^{(1)}=0, \quad \alpha_{3}^{(1)}=-\left(1+p^{2}\right),
$$

$$
\alpha_{4}^{(1)}=0, \quad \alpha_{5}^{(1)}=p q, \quad \alpha_{6}^{(1)}=-p F
$$

$$
\alpha_{1}^{(2)}=0, \quad \alpha_{2}^{(2)}=1+p^{2}, \quad \alpha_{s}^{(2)}=-p q,
$$

$$
\boldsymbol{\alpha}_{4}^{(2)}=p q, \quad \alpha_{5}^{(2)}=1+q^{2}, \quad \alpha_{6}^{(2)}=-q F-p G ;
$$

$$
\boldsymbol{\alpha}_{1}^{(8)}=0, \quad \boldsymbol{\alpha}_{2}^{(3)}=p q, \quad \boldsymbol{\alpha}_{3}^{(8)}=0,
$$

$$
\alpha_{4}^{(8)}=1+q^{2}, \quad \alpha_{5}^{(8)}=0, \quad \alpha_{6}^{(3)}=-q G .
$$

A necessary and sufficient condition that the congruence determined by the straight line be normal is given by the infinite system (32), which here is

$$
\begin{aligned}
& a^{2} \sum A_{i} \alpha_{i}^{(1)}+a b \sum A_{i} \alpha_{i}^{(2)}+b^{2} \sum A_{i} \alpha_{i}^{(8)}=0, \\
& a^{2} \sum B_{i} \alpha_{i}^{(1)}+a b \sum B_{i} \alpha_{i}^{(2)}+b^{2} \sum B_{i} \alpha_{i}^{(3)}=0, \\
& a^{2} \sum C_{i} \alpha_{i}^{(1)}+a b \sum C_{i} \alpha_{i}^{(2)}+b^{2} \sum C_{i} \alpha_{i}^{(3)}=0,
\end{aligned}
$$

If every straight line gives a normal congruence, these equations must subsist for arbitrary values of the ratio $a: b$; we must then have 


$$
\begin{array}{lll}
\sum A_{i} \alpha_{i}^{(1)}=0, & \sum A_{i} \alpha_{i}^{(2)}=0, & \sum A_{i} \alpha_{i}^{(8)}=0, \\
\sum B_{i} \alpha_{i}^{(1)}=0, & \sum B_{i} \alpha_{i}^{(2)}=0, & \sum B_{i} \alpha_{i}^{(8)}=0, \\
\sum C_{i} \alpha_{i}^{(1)}=0, & \sum C_{i} \alpha_{i}^{(2)}=0, & \sum C_{i} \alpha_{i}^{(3)}=0,
\end{array}
$$

Subtracting the derivative of

$$
\sum B_{i} \alpha_{i}^{(1)}=0
$$

from

$$
\sum C_{i} \alpha_{i}^{(1)}=0
$$

we obtain

$$
\sum B_{i} \beta_{i}^{(1)}=0
$$

where the letters $\beta^{(1)}$ are derived from $\alpha^{(1)}$ by the formulas (40), and have the values

$$
\beta_{1}^{(1)}=-\left(1+p^{2}\right), \quad \beta_{2}^{(1)}=-\left(1+p^{2}\right) F_{q}, \quad \beta_{3}^{(1)}=p F-\left(1+p^{2}\right) G_{q}
$$

$$
\begin{gathered}
\beta_{4}^{(1)}=p F-p q F_{q}, \quad \beta_{5}^{(1)}=-q F-p G+p q G_{q}, \\
\beta_{6}^{(1)}=F^{2}+p F_{x}-F_{y}+p G F_{q}-p F G_{q} .
\end{gathered}
$$

The equations

$$
\sum B_{i} \alpha_{i}^{(1)}=0, \quad \sum B_{i} \alpha_{i}^{(2)}=0, \quad \sum B_{i} \alpha_{i}^{(3)}=0,
$$

together with $\left(52^{\prime}\right)$, furnish-when the values of the letters are introduced by means of $(22),\left(49_{1}\right),\left(49_{2}\right),\left(49_{3}\right),\left(53_{1}\right)$-the same system of partial differential equations which have already appeared as (48), (48') and which determined for $F$ and $G$ the forms characteristic of natural families.

If every straight line gives rise to a normal congruence, the family must be natural.

Since the equations (50) are quadratic in $a: b$, if they are satisfied for three values of $a: b$, they are satisfied for all. Therefore, if the straight lines of any triple complex (three lines in each pencil) produce normal congruences the family must be natural.

Any triple complex of curves will serve as well as straight lines. We omit the proof. 
It is sufficient to characterize a family $\infty^{4}$ curves as of the natural type that the two complexes of straight lines parallel to the $x z$ and $x y$ planes, respectively, give rise to normal congruences. For then the equations (50) are satisfied for $a=0$ and for $b=0$. This gives the two infinite systems of equations

$$
\begin{array}{ll}
\sum A_{i} \alpha_{i}^{(1)}=0, & \sum A_{i} \alpha^{(3)}=0, \\
\sum B_{i} \alpha_{i}^{(1)}=0, & \sum B_{i} \alpha_{i}^{(3)}=0, \\
\sum C_{i} \alpha_{i}^{(1)}=0, & \sum C_{i} \alpha_{i}^{(3)}=0,
\end{array}
$$

the analysis of which necessitates for $F$ and $G$ the forms typical of natural families.

Columbia University, New YoRK, N.Y. 